\title{
Review on Pandemic Outbreak of Coronavirus (COVID-19): Virology, Available Treatment, Prevention, and Future Projection
}

\author{
Abhijit Mohan Kanavaje*, Vipul Ajit Sansare
}

\begin{abstract}
Since the outbreak of the novel coronavirus disease COVID-19, caused by the SARS-CoV-2 virus, this disease has spread rapidly around the globe. On 11 March 2020, WHO declared Novel Coronavirus Disease (COVID-19) outbreak as a pandemic and reiterated the call for countries to take immediate actions and scale up the response to treat, detect and reduce transmission to save people's lives. As of 3 April 2020, according to the Ministry of Health \& Family Welfare (MoHFW), a total of 2301 COVID-19 cases (including 55 foreign nationals) have been reported in 29 states/union territories. These include 156 who have been cured/discharged, 1 who has migrated, and 56 deaths in India. Considering the potential threat of a pandemic, scientists and physicians have been racing to understand this new virus and the pathophysiology of this disease to uncover possible treatment regimens and discover effective therapeutic agents and vaccines. The objective of this review article was to have a preliminary opinion about the disease, the ways of treatment, and prevention in this early stage of this outbreak.
\end{abstract}

Keywords: COVID-19, Coronaviruses, Outbreak.

Journal of Applied Pharmaceutical Sciences and Research, (2020); DOI: 10.31069/japsr.v3i1.1

\section{INTRODUCTION}

In December 2019, novel Coronavirus (nCoV), which is another public health problem, has emerged in the Huanan Seafood Market, where livestock animals are also traded, in Wuhan State of Hubei Province in China and has been the focus of global attention due to a pneumonia epidemic of unknown cause. At first, an unknown pneumonia case was detected on December 12, 2019, and possible influenza and other coronaviruses were ruled out by laboratory testing.on $7^{\text {th }}$ January 2020, Chinese authorities announced that a new type of Coronavirus (novel Coronavirus, $\mathrm{nCoV}$ ) was isolated. ${ }^{[1]}$ This virus was named as COVID-19 by WHO on January 12,2020 . As of 3 April 2020, a total of 1,074,290 confirmed cases and 56,987 deaths have been announced. When given where the first case originated, the infection were transmitted probably as zoonotic agent (from animal to human). ${ }^{[2,3]}$

Coronaviruses (CoVs) are the viruses belonging to the Nidovirales order, which includes Coronaviridae, Arteriviridae, and Roniviridae families. The Coronavirinae comprise one of two subfamilies in the Coronaviridae family, with the other being the Torovirinae. The Coronavirinae are further subdivided into four groups, the alpha, beta, gamma and delta coronaviruses. The viruses were initially sorted into these groups based on serology but are now divided by phylogenetic clustering. ${ }^{[4]}$

All viruses in the Nidovirales order are enveloped, nonsegmented positive-sense RNA viruses. They all contain very large genomes for RNA viruses, with Coronavirinae having the largest identified RNA genomes, containing approximately 30 kilobase (kb) genomes. Other common features within the Nidovirales order include: ${ }^{[5,6]}$

- A highly conserved genomic organization, with a large replicase gene preceding structural and accessory genes;

- Expression of many non-structural genes by ribosomal frameshifting;

- Several unique or unusual enzymatic activities encoded within the large replicase-transcriptase polyprotein;

- Expression of downstream genes by a synthesis of 3' nested sub-genomic mRNAs.
Assistant Professor, Indira Institute of Pharmacy, Sadavli, Maharashtra, India

Corresponding Author: A. M. Kanavaje, Assistant Professor, Indira Institute Of Pharmacy, Sadavli, Maharashtra, India Email: amkanavaje@ rediffmail.com

How to cite this article: Kanavaje AM, Sansare VA. Review on Pandemic Outbreak Of Coronavirus (COVID-19): Virology, Available Treatment, Prevention, and Future Projection. Journal of Applied Pharmaceutical Sciences and Research, 2020; 3(1):1-6

Source of support: Nil

Conflict of interest: None

\section{Virology-Pathogenesis}

Coronavirus virions are spherical with diameters of approximately $125 \mathrm{~nm}$, as depicted in recent studies by cryo-electron tomography and cryo-electron microscopy. The most prominent feature of coronaviruses is the club-shaped spike projections emanating from the surface of the virion. These spikes are a defining feature of the virion and give them the appearance of a solar corona, prompting the name, coronaviruses. ${ }^{[7]}$

As mentioned earlier, coronaviruses contain a non-segmented, positive-sense RNA genome of $\sim 30 \mathrm{~kb}$. For the translation of the replicase polyproteins, the genome contains a 5 cap structure along with a 3 ' poly (A) tail, allowing it to act as a mRNA. Coronaviruses are viruses whose genome structure is best known among all RNA viruses. Two-thirds of RNA they have encodes viral polymerase (RdRp), RNA synthesis materials, and two large nonstructural polyproteins that are not involved in host response modulation. The other one-third of the genome encodes four structural proteins spike (S), envelope (E), membrane (M) ve nucleocapsid(N), and the other helper proteins. ${ }^{[5,7,8]}$

The trimetric $S$ glycoprotein is a class I fusion protein and mediates attachment to the host receptor. S1 makes up the large receptor-binding domain of the S protein, while S2 forms the stalk of the spike molecule.

The $\mathrm{M}$ protein is the most abundant structural protein in the virion. It is a small $(\sim 25-30 \mathrm{kDa})$ protein with three transmembrane 
domains. It has a small $\mathrm{N}$-terminal glycosylated ectodomain and a much larger C-terminal endo-domain that extends 6-8 $\mathrm{nm}$ into the viral particle and is thought to give the virion its shape. The structure of Corona virus is shown in Figure 1.

The $\mathrm{N}$ protein constitutes the only protein present in the nucleocapsid. It is composed of two separate domains, an $\mathrm{N}$-terminal domain (NTD) and a C-terminal domain (CTD), both capable of binding RNA in vitro, but each domain uses different mechanisms to bind RNA. The E protein $(\sim 8-12 \mathrm{kDa})$ is found in small quantities within the virion. E protein from coronaviruses are highly divergent but have a common architecture. The replicase gene encoding the nonstructural proteins (Nsps) occupies twothirds of the genome, about $20 \mathrm{~kb}$, as opposed to the structural and accessory proteins, which make up only about $10 \mathrm{~kb}$ of the viral genome. The functions of various nonstructural proteins are tabulated in Table 1 . The $5^{\prime}$ end of the genome contains a leader sequence and untranslated region (UTR) that contains multiple stem-loop structures required for RNA replication and transcription..$^{[3,5,8,9]}$

\section{Life Cycle Of Coronavirus}

\section{Attachment and Entry}

The initial attachment of the virion to the host cell is initiated by interactions between the $S$ protein and its receptor. The sites of receptor binding domains (RBD) within the S1 region of a coronavirus $S$ protein vary depending on the virus, with some having the RBD at the N-terminus of S1 (MHV) while others (SARS$\mathrm{CoV}$ ) have the RBD at the C-terminus of S1. The S-protein/receptor

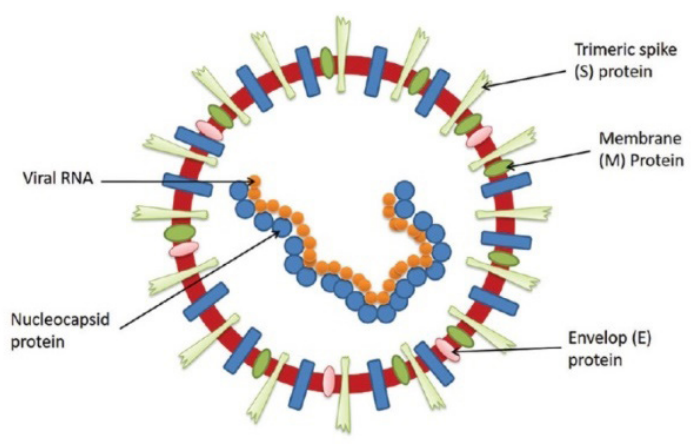

Figure 1: Structure of coronavirus ${ }^{[5]}$

interaction is the primary determinant for a coronavirus to infect a host species and also governs the tissue tropism of the virus. ${ }^{[9,11]}$ Following receptor binding, the virus must next gain access to the host cell cytosol. This is generally accomplished by acid-dependent proteolytic cleavage of S protein by a cathepsin, TMPRRS2, or another protease, followed by fusion of the viral and cellular membranes. The formation of this bundle allows for the mixing of viral and cellular membranes, resulting in fusion and ultimately release of the viral genome into the cytoplasm. ${ }^{[9-11]}$

\section{Replicase Protein Expression}

The next step in the coronavirus lifecycle is the translation of the replicase gene from the virion genomic RNA. The replicase gene encodes two large ORFS, rep1a and rep1b, which express

\section{Attachment and Entry

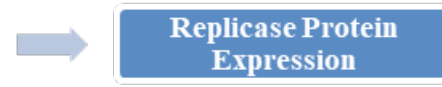

Table 1: Functions of coronavirus nonstructural proteins $(\mathrm{nsps})^{[3]}$

\begin{tabular}{ll}
\hline Protein & Function \\
\hline nsp1 & Promotes cellular mRNA degradation and blocks host-cell translation, results in blocking innate immune response \\
nsp2 & No known function binds to prohibitin proteins \\
nsp3 & Large, multi-domain transmembrane protein, activities include: \\
& Ubl1 and Ac domains, interact with N protein \\
& ADRP activity promotes cytokine expression \\
& PLPro/Deubiquitinase domain cleaves viral polyprotein and blocks host innate immune response \\
& Ubl2, NAB, G2M, SUD, Y domains, unknown functions \\
nsp4 & Potential transmembrane scaffold protein, important for proper structure of DMVs \\
nsp5 & Mpro, cleaves viral polyprotein \\
nsp6 & Potential transmembrane scaffold protein \\
nsp7 & Forms hexadecameric complex with nsp8, may act as processivity clamp for RNA polymerase \\
nsp8 & Forms hexadecameric complex with nsp7, may act as processivity clamp for RNA polymerase; may act asprimase \\
nsp9 & RNA binding protein \\
nsp10 & Cofactor for nsp16 and nsp14, forms a heterodimer with both and stimulates ExoN and 2-O-MT activity \\
nsp12 & RdRp \\
nsp13 & RNA helicase, 5区 triphosphatase \\
nsp14 & N7 MTase) and 3区-5区 exoribonuclease, ExoN; N7MTase adds 5区 cap to viral RNAs, ExoN activity is important for proofreading of \\
& the viral genome \\
nsp15 & Viral endoribonuclease, NendoU \\
nsp16 & 2'-O-MT; shields viral RNA from MDA5 recognition \\
\hline
\end{tabular}

(Ubl, ubiquitin-like; Ac, acidic; ADRP, ADP-ribose-1'-phosphate; PLPro, papain-like protease; NAB, nucleic acid binding; SUD, SARS-unique domain; DMVs, double-membrane vesicles; Mpro, main protease; RdRp, RNA-dependent RNA polymerase; MTase, methyltransferase; Viral exoribonuclease, ExoN; Viral endoribonuclease, NendoU; 2'-O-MT, 2'-O-methyltransferase; MDA5, Melanoma differentiation-associated protein 5) ${ }^{[6,10]}$ 
two co-terminal polyproteins, pp1a and pp1ab. In most cases, the ribosome unwinds the pseudoknot structure and continues translation until it encounters the rep1a stop codon. Occasionally the pseudoknot blocks the ribosome from continuing elongation, causing it to pause on the slippery sequence, changing the reading frame by moving back one nucleotide, -1 frameshift, before the ribosome is able to melt the pseudoknot structure and extend translation into rep $1 \mathrm{~b}$, resulting in the translation of pplab. In vitro studies predict the incidence of ribosomal frameshifting to be as high as $25 \%$, but this has not been determined in the context of virus infection. Next, many of the nsps assemble into the replicasetranscriptase complex (RTC) to create an environment suitable for RNA synthesis, and ultimately are responsible for RNA replication and transcription of the sub-genomic RNAs. ${ }^{[9]}$

\section{Replication and Transcription}

Viral RNA synthesis follows the translation and assembly of the viral replicase complexes. Viral RNA synthesis produces both genomic and sub-genomic RNAs. Sub-genomic RNAs serve as mRNAs for the structural and accessory genes which reside downstream of the replicase polyproteins. All positive-sense sub-genomic RNAs are $3^{\prime}$ co-terminal with the full-length viral genome and thus form a set of nested RNAs, a distinctive property of the order Nidovirales.

Perhaps the most novel aspect of coronavirus replication is how the leader and body TRS segments fuse during the production of sub-genomic RNAs. This was originally thought to occur during positive-strand synthesis, but now it is largely believed to occur during the discontinuous extension of negative-strand RNA. The current model proposes that the RdRp pauses at any one of the body TRS sequences (TRS-B). Following this pause, the RdRp either continues elongation to the next TRS, or it switches to amplifying the leader sequence at the $5^{\prime}$ end of the genome-guided by the complementarity of the TRS-B to the leader TRS (TRS-L). Many pieces of evidence currently support this model, including the presence of anti-leader sequence at the $3^{\prime}$ end of the negative-strand subgenomic RNAs. Finally, coronaviruses are also known for their ability to recombine using both homologous and non-homologous recombination. The ability of these viruses to recombine is tied to the strand switching ability of the RdRp. Recombination likely plays a prominent role in viral evolution and is the basis for targeted RNA recombination, a reverse genetics tool used to engineer viral recombinants at the $3^{\prime}$ end of the genome..$^{[3,9,11]}$

\section{Assembly and Release}

Following replication and subgenomic RNA synthesis, the viral structural proteins, $\mathrm{S}, \mathrm{E}$, and $\mathrm{M}$ are translated and inserted into the endoplasmic reticulum (ER). These proteins move along the secretory pathway into the endoplasmic reticulum-Golgi intermediate compartment (ERGIC). There, viral genomes encapsidated by $\mathrm{N}$ protein bud into membranes of the ERGIC containing viral structural proteins, forming mature virions.

The $M$ protein directs most protein-protein interactions required for assembly of coronaviruses. $N$ protein enhances VLP formation, suggesting that the fusion of encapsidated genomes into the ERGIC enhances viral envelopment. The $S$ protein is incorporated into virions at this step but is not required for assembly. While the $M$ protein is relatively abundant, the $E$ protein is only present in small quantities in the virion. It is unknown how $E$ protein assists $M$ protein in assembly of the virion, and several possibilities have been suggested. The E protein may also have a separate role in promoting viral release by altering the host secretory pathway. The $\mathrm{M}$ protein also binds to the nucleocapsid, and this interaction promotes the completion of virion assembly. ${ }^{[3,5,9,12]}$ The lifecycle of Corona virus is depicted in Figure 2.

\section{Sources and Modes of Transmission}

Coronaviruses cause a large variety of diseases in animals, and their ability to cause severe disease in livestock and companion animals such as pigs, cows, chickens, dogs, and cats led to significant research on these viruses in the last half of the 20th century. CoVs have been defined as a novel respiratory tract virus in the samples collected from the individuals who present symptoms of respiratory tract infection. ${ }^{[4]}$

The most heavily studied animal coronavirus is murine hepatitis virus (MHV), which causes a variety of outcomes in mice, including respiratory, enteric, hepatic, and neurologic infections. These infections often serve as highly useful models of disease. For instance, MHV-1 causes severe respiratory disease in susceptible $\mathrm{A} / \mathrm{J}$ and $\mathrm{C} 3 \mathrm{H} / \mathrm{HeJ}$ mice, $\mathrm{A} 59$ and MHV-3 induce severe hepatitis, while JHMV causes severe encephalitis. Interestingly, MHV-3 induces cellular injury through the activation of the coagulation cascade. Most notably, A59 and attenuated versions of JHMV cause a chronic demyelinating disease that bears similarities to multiple sclerosis (MS), making MHV infection one of the best models for this debilitating human disease. Early studies suggested that demyelination was dependent on viral replication in oligodendrocytes in the brain and spinal cord; however, more recent reports clearly demonstrate that the disease is immune-mediated. Irradiated mice or immunodeficient (lacking T and B cells) mice do not develop demyelination, but addition of virus-specific $T$ cells restores the development of demyelination. ${ }^{[3,14,15]}$

Prior to the SARS-CoV outbreak, coronaviruses were only thought to cause mild, self-limiting respiratory infections in humans. Two of these human coronaviruses are a-coronaviruses (HCoV-229E and HCoV-NL63) while the other two are $\beta$-coronaviruses (HCoVOC43 and HCoV-HKU1). HCoV-229E and HCoV-OC43 were isolated nearly 50 years ago, while HCoV-NL63 and HCoV-HKU1 were only recently identified following the SARS-CoV outbreak. These viruses are endemic in the human population, causing $15-30 \%$ of respiratory tract infections each year. They cause more severe diseases in neonates, the elderly, and individuals with underlying illnesses, with a greater incidence of lower respiratory tract infection in these populations. ${ }^{[4]}$

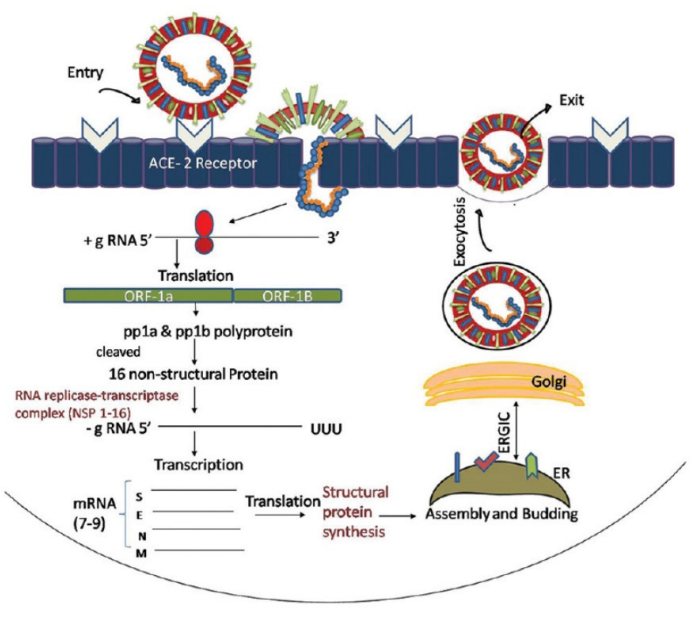

Figure 2: Life Cycle of CoV in a host cell ${ }^{[5]}$ 
SARS-CoV, a group $2 b \beta$-coronavirus, was identified as the causative agent of the Severe Acute Respiratory Syndrome (SARS) outbreak that occurred in 2002-2003 in the Guangdong Province of China. It is the most severe disease caused by any coronavirus. During the 2002-2003 outbreak, approximately 8098 cases occurred with 774 deaths, resulting in a mortality rate of $9 \%$. During the epidemic, closely related viruses were isolated from several exotic animals, including Himalayan palm civets and raccoon dogs. However, it is widely accepted that SARS-CoV originated in bats as a large number of Chinese horseshoe bats contain sequences of SARS-related CoVs and contain serologic evidence for prior infection with a related CoV. Two novel bat SARS-related CoVs were recently identified that are more similar to SARS-CoV than any other virus identified to date. They were also found to use the same receptor as the human virus, angiotensin-converting enzyme 2 (ACE2), providing further evidence that SARS-CoV originated in bats. Although some human individuals within wet animal markets, had serologic evidence of SARS-CoV infection before the outbreak, these individuals had no apparent symptoms.

The transmission of SARS-CoV was relatively inefficient, as it only spread through direct contact with infected individuals after the onset of illness. Thus, the outbreak was contained mainly within households and healthcare settings, except in a few cases of superspreading events where one individual was able to infect multiple contacts due to enhanced development of high viral burdens or ability to aerosolize virus. As a result of the relatively inefficient transmission of SARS-CoV, the outbreak was controllable through the use of quarantine.

SARS-CoV primarily infects epithelial cells within the lung. The virus is capable of entering macrophages and dendritic cells but only leads to an abortive infection. Despite this, infection of these cell types may be important in inducing pro-inflammatory cytokines that may contribute to disease.The exact mechanism of lung injury and cause of severe disease in humans remains undetermined. Viral titers seem to diminish when severe disease develops in both humans and in several animal models of the disease. While the SARS-CoV epidemic was controlled in 2003 and the virus has not since returned, a novel human CoV emerged in the Middle East in 2012. This virus, named Middle East Respiratory Syndrome-CoV (MERS-CoV), was found to be the causative agent in a series of highly pathogenic respiratory tract infections in Saudi Arabia and other countries in the Middle East. Before SARS-CoV cases, it was thought that human CoVs leads to cold-like upper respiratory infection and self-limiting lower respiratory infection. The first death due to coronaviruses has reported by the isolation of SARS-CoV from a patient with pneumonia in China. As in other respiratory infected viruses and previous beta-CoV, similarities present in the clinical aspects of COVID-19 infections, it is known that clinical picture varies from simple respiratory infection findings to septic shock. Similar to SARS CoV and MERS CoV that caused epidemics in the past years, the first symptoms are commonly defined as fever, cough, shortness of breath. ${ }^{[14,15]}$

\section{Treatment and Protection}

To date, there are no anti-viral therapeutics that specifically target human coronaviruses, so treatments are only supportive. In vitro, interferons (IFNs) are only partially effective against coronaviruses. In general, there are few or no treatment options for viral diseases that occur suddenly. In parallel with this knowledge, today, there is no vaccine or effective treatment to prevent COVID-19 infection. Molecules are being tested for COVID-19 in in-vitro and human- based SARS-CoV and MERS-Cov trials. The SARS and MERS outbreaks have stimulated research on these viruses, and this research has identified a large number of suitable anti-viral targets, such as viral proteases, polymerases, and entry proteins. Significant work remains, however, to develop drugs that target these processes and are able to inhibit viral replication. Only limited options are available to prevent coronavirus infections. Studies evaluating the antiviral activity of types I and II interferons have reported, interferon-beta (IFNb), as the most potent interferon, was reducing in- vitro MERS$\mathrm{CoV}$ replication. According to a human MERSCoV case report from South Korea, the use of the combination of Lopinavir/Ritonavir (LPV/ RTV) (Anti-HIV drugs), pegylated interferon and ribavirin provided a successful viral clearance. For this purpose, a randomized control trial (MIRACLE Trial), that aimed to determine whether LPV/RTV-IFNb improved clinical results in MERS-CoV patients. Although another antiviral drug, Remdesivir, was used in the first case reported from the United States of America, it seemed successful. In-vitro studies have shown that viral RNA transcription was terminated with Remdesivir in an early stage. Publications are demonstrating that remdesivir has a strong antiviral activity in epithelial cell cultures against SARS-CoV, MERS-CoV, and related zoonotic bat CoVs.Many measures should be taken, such as timely publication of epidemic information for the elimination of the source of infection, early diagnosis, reporting, isolation, supportive treatments, and for avoiding unnecessary panic. CDC reminds necessary measures such as hand washing, using disinfectant solutions, avoiding contact with patients to prevent the spread of viruses by droplets. Precautionary actions, including the provision of medicines supply chains, personal protective equipment, and hospital supplies, should be made in a short time for the protection of the Chinese people and global health, especially in places with close travel ports to major Chinese ports. Based on the 2003 SARS-CoV epidemic experience, the government takes many effective measures, including closing public transport, reducing migration, and promoting personal protection with masks. Healthcare staff should be informed about taking personal protective measures such as the use of gloves, eye masks, and $\mathrm{N} 95$ masks during the examination of patients with a suspected history of COVID-19. ${ }^{[3,6,14,15]}$

\section{Future Projections}

The coronavirus COVID-19 is affecting 204 countries and territories around the world and two international conveyances. There are currently 1,074,290 confirmed cases and 56,987 deaths from the coronavirus COVID-19 outbreak as of April 03, 2020. Since COVID19 is very similar to SARS-CoV, some important features of SARS epidemic are guiding the predictions on the current epidemic. According to the logistical modeling studies performed by combining daily numbers from COVID-19 cases with data obtained in SARS epidemics; timely diagnosis is essential for quarantine and integrated interventions to control the outbreak. Currently, growth factor (New cases of everyday/cases of the previous day) of COVID-19 started to fall below one threshold. If the current trend continues, the number of infected people is expected to reach peak at the beginning of March 2020(80 days from the onset). The duration between onset of symptoms and isolation is about 6 days, and it's expected that each one-day reduction in this period will decrease the size of peak population by $72-84 \%$ and cumulative infected cases and deaths by $68-80 \%$. It is estimated that with the effects of integrated interventions such as promoting the use of face masks and reduced traveling, each $10 \%$ reduction in transmission rate, the size of peak population will decrease by $20-47 \%$, and 
Table 2: The Number of Cases and Death of COVID-19 outbreak according to the World Health Organization (WHO) Situation Reports-22 on April 3, 2020. ${ }^{[17]}$

\begin{tabular}{|c|c|c|c|c|c|c|c|c|}
\hline $\begin{array}{l}\text { Country, } \\
\text { Other }\end{array}$ & $\begin{array}{l}\text { Total } \\
\text { Cases }\end{array}$ & $\begin{array}{l}\text { New } \\
\text { Cases }\end{array}$ & $\begin{array}{l}\text { Total } \\
\text { Deaths }\end{array}$ & $\begin{array}{l}\text { Total } \\
\text { Recovered }\end{array}$ & $\begin{array}{l}\text { Active } \\
\text { Cases }\end{array}$ & $\begin{array}{l}\text { Serious, } \\
\text { Critical }\end{array}$ & $\begin{array}{l}\text { Tot Case/ } \\
1 M \text { pop }\end{array}$ & $\begin{array}{l}\text { Deaths/ } \\
\text { 1M pop }\end{array}$ \\
\hline World & $1,074,290$ & $+59,225$ & 56,987 & 226,062 & 791,241 & 38,973 & 137.8 & 7.3 \\
\hline USA & 265,506 & $+20,629$ & 6,786 & 11,983 & 246,737 & 5,781 & 802 & 21 \\
\hline Italy & 119,827 & $+4,585$ & 14,681 & 19,758 & 85,388 & 4,068 & 1,982 & 243 \\
\hline Spain & 117,710 & $+5,645$ & 10,935 & 30,513 & 76,262 & 6,416 & 2,518 & 234 \\
\hline Germany & 89,838 & $+5,044$ & 1,230 & 24,575 & 64,033 & 3,936 & 1,072 & 15 \\
\hline China & 81,620 & +31 & 3,322 & 76,571 & 1,727 & 379 & 57 & 2 \\
\hline France & 59,105 & & 5,387 & 12,428 & 41,290 & 6,399 & 905 & 83 \\
\hline Iran & 53,183 & $+2,715$ & 3,294 & 17,935 & 31,954 & 4,035 & 633 & 39 \\
\hline UK & 38,168 & $+4,450$ & 3,605 & 135 & 34,428 & 163 & 562 & 53 \\
\hline Turkey & 20,921 & $+2,786$ & 425 & 484 & 20,012 & 1,251 & 248 & 5 \\
\hline Switzerland & 19,606 & +779 & 591 & 4,846 & 14,169 & 348 & 2,265 & 68 \\
\hline Belgium & 16,770 & $+1,422$ & 1,143 & 2,872 & 12,755 & 1,205 & 1,447 & 99 \\
\hline Netherlands & 15,723 & $+1,026$ & 1,487 & 250 & 13,986 & 1,182 & 918 & 87 \\
\hline Canada & 11,747 & +464 & 173 & 1,979 & 9,595 & 120 & 311 & 5 \\
\hline Austria & 11,489 & +360 & 168 & 2,022 & 9,299 & 245 & 1,276 & 19 \\
\hline S. Korea & 10,062 & +86 & 174 & 6,021 & 3,867 & 55 & 196 & 3 \\
\hline Portugal & 9,886 & +852 & 246 & 68 & 9,572 & 245 & 970 & 24 \\
\hline Brazil & 8,229 & +185 & 343 & 127 & 7,759 & 296 & 39 & 2 \\
\hline Israel & 7,030 & +173 & 40 & 338 & 6,652 & 115 & 812 & 5 \\
\hline Sweden & 6,131 & +563 & 358 & 205 & 5,568 & 469 & 607 & 35 \\
\hline Australia & 5,350 & +36 & 28 & 585 & 4,737 & 85 & 210 & 1 \\
\hline Norway & 5,296 & +149 & 57 & 32 & 5,207 & 96 & 977 & 11 \\
\hline Ireland & 4,273 & +424 & 120 & 5 & 4,148 & 109 & 865 & 24 \\
\hline Russia & 4,149 & +601 & 34 & 281 & 3,834 & 8 & 28 & 0.2 \\
\hline Czechia & 4,091 & +233 & 53 & 72 & 3,966 & 77 & 382 & 5 \\
\hline Denmark & 3,757 & +371 & 139 & 1,193 & 2,425 & 153 & 649 & 24 \\
\hline Chile & 3,737 & +333 & 22 & 427 & 3,288 & 31 & 195 & 1 \\
\hline Ecuador & 3,368 & +205 & 145 & 65 & 3,158 & 100 & 191 & 8 \\
\hline Malaysia & 3,333 & +217 & 53 & 827 & 2,453 & 108 & 103 & 2 \\
\hline Poland & 3,266 & +320 & 65 & 56 & 3,145 & 50 & 86 & 2 \\
\hline Romania & 3,183 & +445 & 133 & 283 & 2,767 & 83 & 165 & 7 \\
\hline Philippines & 3,018 & +385 & 136 & 52 & 2,830 & 1 & 28 & 1 \\
\hline Pakistan & 2,637 & +216 & 40 & 126 & 2,471 & 10 & 12 & 0.2 \\
\hline Japan & 2,617 & & 63 & 514 & 2,040 & 60 & 21 & 0.5 \\
\hline Luxembourg & 2,612 & +125 & 31 & 500 & 2,081 & 33 & 4,173 & 50 \\
\hline India & 2,567 & +24 & 72 & 192 & 2,303 & & 2 & 0.05 \\
\hline
\end{tabular}

cumulative infected cases and deaths will decrease by $23-49 \%$. The number of COVID19 infections and deaths as on April 3, 2020 in different countries is presented in Table 2. Owing to the measures by the government, including passing laws for effective infection management, supports accelerating the diagnosis and treatment such as the distribution of more than 30.000 PCR-fluorescent probe kits to determined diagnosis centers in the country, the number of cases are expected to be below the estimates. Rapid diagnosis with quarantine and integrated interventions will have a great effect on future trends of the outbreak. Although COVID- 19 has a similar spread with SARS and MERS, it exhibits lower mortality rates. As the imposition of globalization, coronaviruses will cause spreads and outbreaks with different-mutant strains similarly in the coming years. With increased scientific collaboration, which is a result of globalization, we may have more powerful means of fighting against coronaviruses, in which we know the genome structure very well in the future. Future research on coronaviruses will continue to investigate many aspects of viral replication and pathogenesis. First, understanding the propensity of these viruses to jump between species, to establish infection in a new host, and to identify significant reservoirs of coronaviruses will dramatically aid in our ability to predict when and where potential epidemics may occur. As bats seem to be a significant reservoir for these viruses, it will be interesting to determine how they seem to avoid clinically evident disease and become persistently infected. Second, many of the non-structural and accessory proteins encoded by these viruses remain uncharacterized with no known function, and it will be important to identify mechanisms of action for these proteins as well as defining their role in viral replication and pathogenesis. These studies should lead to a large increase in the number of suitable therapeutic targets to combat infections. ${ }^{[2,16]}$ 


\section{Conclusion}

Owing to the lack of effective therapeutics or vaccines, the best measures to control human coronaviruses remain a strong public health surveillance system coupled with rapid diagnostic testing and quarantine when necessary. For international outbreaks, cooperation of governmental entities, public health authorities, and health care providers is critical. During veterinary outbreaks that are readily transmitted, such as PEDV, more drastic measures such as destruction of entire herds of pigs may be necessary to prevent transmission of these deadly viruses. Finally, defining the mechanism of how coronaviruses cause disease and understanding the host immunopathological response will significantly improve our ability to design vaccines and reduce disease burden.

\section{References}

1. Egloff MP, Ferron F, Campanacci V, Longhi S, Rancurel C, Dutartre H, Snijder EJ, Gorbalenya AE, Cambillau C, Canard B. The severe acute respiratory syndrome-coronavirus replicative protein nsp9 is a single-stranded RNA-binding subunit unique in the RNA virus world. Proceedings of the National Academy of Sciences of the United States of America. 2004; 101(11):3792-3796. [PubMed: 15007178]

2. https://www.who.int/emergencies/diseases/novel-coronavirus-2019/ situation-reports/

3. World Helath Organization, 2019- nCoV Situation Report-22 on 12 February, 2020. https://www.who.int/docs/defaultsource/ coronaviruse/situation-reports/

4. Yin Y, Wunderink RG. MERS, SARS and other corona viruses as causes of pneumonia. Respirology 2018;23:130-7.

5. Prajapat M,Saram P,Shekhar N,Avti P et.al,Drug Target For Corona Virus: A Systematic Review. Indian J Pharmacol 2020;52:56-65.

6. Anthony R. Fehr and Stanley Perlman Coronaviruses: An Overview of Their Replication and Pathogenesis 2015 ; 1282: 1-23. doi:10.1007/978-
1-4939-2438-7_1

7. Bosch BJ, van der Zee R, de Haan CA, Rottier PJ. The coronavirus spike protein is a class I virus fusion protein: structural and functional characterization of the fusion core complex. J Virol. 2003; 77(16):88018811. [PubMed: 12885899]

8. Pavan K. Bhatraju, M.D., Bijan J.Covid-19 in Critically III Patients in the Seattle Region - Case Series The New England Journal of Medicine,

9. Ahmet Riza Sahin, Aysegul Erdogan,2019 Novel Coronavirus (COVID19) Outbreak:A Review of the Current Literature DOI: 10.14744/ ejmo.2020.12220EJMO 2020;4(1):1-7

10. Imbert I, Guillemot JC, Bourhis JM, Bussetta C, Coutard B, Egloff MP, Ferron F, Gorbalenya AE, Canard B. A second, non-canonical RNAdependent RNA polymerase in SARS coronavirus. EMBO J. 2006; 25(20):4933-4942. [PubMed: 17024178]

11. Zhai Y, Sun F, Li X, Pang H, Xu X, Bartlam M, Rao Z. Insights into SARS$\mathrm{CoV}$ transcription and replication from the structure of the nsp7-nsp8 hexadecamer. Nat Struct Mol Biol. 2005; 12(11):980-986. [PubMed: 16228002].

12. Gralinski L, Menachery V. Return of the Coronavirus: 2019-nCoV, Viruses 2020;12:135.

13. What to do if you are sick with 2019 Novel Coronavirus. Available online:https://www.cdc.gov/coronavirus/2019-ncov/about/stepswhen-sick.html (accessed on 05 February 2020)

14. 2019 Novel Coronavirus.Prevention and Treatment. Available online: https://www.cdc.gov/coronavirus/2019-ncov/about/ preventiontreatment.html (accessed on 05 February 2020)

15. World Health Organization. Novel coronavirus-China. January 12, 2020 (http://www.who.int/csr/don/12-january-2020-novelcoronavirus -china/en/).

16. A novel coronavirus outbreak of global health concern - Chen Want et al. The Lancet. January 24,2020

17. Chang CK, Sue SC, Yu TH, Hsieh CM, Tsai CK, Chiang YC, Lee SJ, Hsiao $\mathrm{HH}$, Wu WJ, Chang WL, Lin CH, Huang TH. Modular organization of SARS coronavirus nucleocapsid protein. Journal of biomedical science. 2006; 13(1):59-72. [PubMed: 16228284] 